\title{
Sinus of Valsalva
}

National Cancer Institute

\section{Source}

National Cancer Institute. Sinus of Valsalva. NCI Thesaurus. Code C33557.

Any one of the three normal anatomical dilatations of the aortic wall, which are located behind each of the cusps of the aortic valve. 